\title{
CLONING OF POTENTIAL CANDIDATES FOR GUINEA PIG OPIOID RECEPTORS
}

\author{
J. Fickel, G. Xie, R. C. Thompson, S. J. Watson and H. Akil, Mental \\ Health Research Institute, University of Michigan, Ann Arbor, MI 48109
}

The endogenous opioid system plays an important role in modulating endocrine, respiratory, cardiovascular and immune functions and others (1). Opioids exert their actions by binding to specific, G-protein coupled seven-transmembrane domaine receptors, which have been classified by their ligands as delta, mu, and kappa. Based on the recently cloned mouse delta opioid receptor DNA sequence $(2,3)$, other opioid receptors were also successfully cloned $(4,5)$. In light of the recent cloning and pharmacological characterization of the guinea pig (gp) kappa receptor in our lab (4), the aim of this work was to clone and characterize the gp brain delta- and mu receptors. We were able to clone a 675 bp guinea pig mu opioid receptor sequence, corresponding to the AA 153-383 of the rat mu opioid receoptor (5). Additionally we were able to clone a $571 \mathrm{bp}$ fragment, homologous to the AA 138-329 of the mouse delta opioid receptor $(1,2)$.

Methods: a) cDNA library construction and screening: we used a Lambda gt11 gp brain cDNA library (Clonetech) with 1.3 million independent clones, oligo(dT)-primed, cDNA's inserted into the EcoRI site of Lambda gt11 and ranging in size from $1.3 \mathrm{~kb}$ to $4 \mathrm{~kb}$. For new libraries we used gp brain mRNAs to construct several cDNA libraries in the following vectors: pME18SNeo, pME18S, pcD-Neo-SRa-X. b) PCR: P1: 5'-CTCACCATGATGAGCG TGGA-3'; P2: 5'-AGCAGCGCTTGAAGTTCTCG-3', P3: 5'-TCGATCCACTGTATTAGCCG-

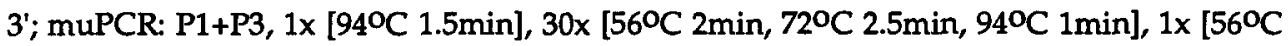

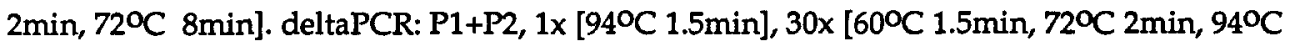
$1 \mathrm{~min}], 1 \times\left[60^{\circ} \mathrm{C} 1.5 \mathrm{~min}, 72^{\circ} \mathrm{C} 6 \mathrm{~min}\right.$ ]. The resulting PCR fragments were isolated and finally cloned into pBSSKIII+ (Stratagene). After transformation into E.coli and colony screening, the plasmids from positive clones were isolated, and both strands were sequenced using the PCR primer and the plasmid T7- and T3 RNA polymerase promoter primer. Results: The isolated DNAs from those $\mathrm{gp}$ cDNA libraries (including the Lambda gt11 gp brain cDNA library) have been hybridized with a rat delta opioid receptor (470bp, part of the ORF) and a rat mu opioid receptor probe (1.4kb, containing the complete ORF). All cDNA libraries gave positive signals with both probes and were subsequently used for the PCR reactions. Using the sequence analysis software GCG (University Wisconsin) we translated both sequences into open reading frames and aligned them to the rat and guinea pig kappa-, the rat mu- and the mouse delta opioid receptor sequences. The results are shown in Fig. 1.

Fig.1 Aminoacid-alignement of the cloned gP mu- and delta opioid receptor sequences

1

Rk ..........MESPIQIF RGEPGPTCAP SACLLPNSSS WFPNWAESDS NGSVGSEDQQ

Gk ......... MGRRRQGP AQPASELPAR NACLLPNGSA WLPGWAEPDG NGSAGPQDEQ

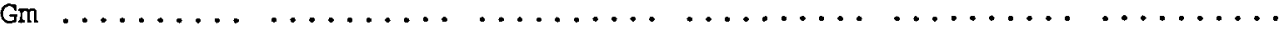

RI MDSSTGPGNT SDCSDPLAQA SCSPAPGSWL NLSHVDGNQS DPCGLNRTGL GGNDSLCPQT

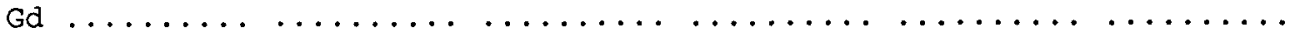

Md .........ME LV P SARA ELQSS PLVNL SD AFPSAFPSAG ANASGSPGAR

61

TM-I

RK LEPAHISPAI PVIITAVYSV VFVVGLVGNS LVMFVIIRYT KMKTATNIYI FNLALADALV

Gk LEPAHISPAI PVIITAVYSV VFVVGLVGNS LVMFVIIRYT KMKTATNIYI FNLALADALV

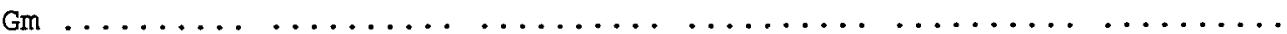

RM GSP...SMVT AITIMALYSI VCVVGLFGNF LVMYVIVRYT KMKTATNIYI FNLALADALA

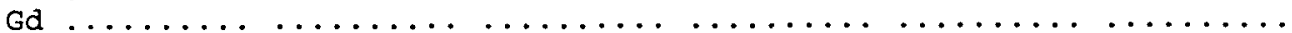

Md SAS...SLAL AIAITALYSA VCAVGLLGNV LVMFGIVRYT KLKTATNIYI FNLALADALA 
Rk TTTMPFQSAV YLMNSWPFGD VLCKIVISID YYNMFTSIFT LTMMSVDRYI AVCHPVKALD

Gk TTTMPFQSTV YLMNSWPFGD VLCKIVISID YYNMFTSIFT LTMMSVDRYI AVCHPVKALD

$\mathrm{Gm} \ldots \ldots \ldots \ldots \ldots \ldots \ldots \ldots \ldots \ldots \ldots \ldots \ldots \ldots$ LTMSVDRYI AVCHPVKALD

RM TSTLPFQSVN YLMGTWPFGT ILCKIVISID YYNMFTSIFT LCTMSVDRYI AVCHPVKALD

Gd $\ldots \ldots \ldots \ldots \ldots \ldots \ldots \ldots \ldots \ldots \ldots \ldots \ldots \ldots \ldots$ LTMMSVDRYI AVCHPVKALD

Md TSTLPFQSAK YLMETWPFGE LLCKAVISID YYNMFTSIFT LTMMSVDRYI AVCHPVKALD 181

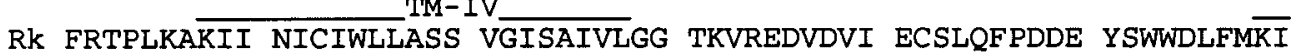

Gk FRTPLKAKII NICIWLLSSS VGISAIILGG TKVREDVDII ECSLQFPDDD YSWWDLFMKI

GM FRTPRNAKIV NVCNWILSSA IGLPVMFMAT TKYRQGS..I DCTLTFSHPT W.YWENLLKI

RM FRTPRNAKIV NVCNWILSSA IGLPVMFMAT TKYRQGS.. I DCTLTFSHPT $W$.YWENLLKI

Gd FRTPAKAKLI NICIWVLASG VGVPIMVMAV TQPRDGA..V VCTLQFPSPS $W$.YWDTVTKI

Md FRTPAKAKLI NICIWVLASG VGVPIMVMAV TQPRDGA..V VCMLQFPSPS $W$.YWDTVTKI 241

$T M-V$ TM-VI

Rk CVFVFAFVIP VLIIIVCYTI MILRLKSVRL LSGSREKDRN LRRITKLVLV VVAVFIICWT

GK CVFVFAFVIP VLIIIVCYTL MILRLKSVRL LSGSREKDRN LRRITRLVLV VVAVFIICWT

GM CVFIFAFIMP VLIITVCYGL MILRLKSVRM LSGSKEKDRN LRRITRMVLV VVAVFIVCWT

RM CVFIFAFIMP VLIITVCYGL MILRLKSVRM LSGSKEKDRN LRRITRMVLV VVAVFIVCWT

Gd CVFLFAFVVP TLIITVCYGL MLIRLRSVRL LSGSKEKDRS LRRITRMVLV VVGAFVVCWA

Md CVFLFAFVVP ILIITVCYGL MLLRLRSVRL LSGSKEKDRS LRRITRMVLV VVGAFVVCWA 301

TM-VII

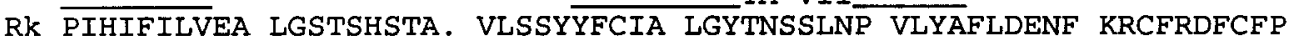

GK PIHIFILVEA LGSTSHSTA. ALSSYYFCIA LGYTNSSLNP ILYAFLDENF KRCFRDFCFP

GM PIAIYVIIKA LITI.PETTF QTVSWHFCIA LGYTNSCLNP VLYAFLDENF KRCFREFCIP

RM PIHIYVIIKA LITI. PETTF QTVSWHFCIA LGYTNSCLNP VLYAFLDENF KRCFREFCIP

Gd PIHIFVIVWT LVDINRRDPL VVAALHLCIA LAYANSSLNP VLYAFLDENF KRC

Md PIHIFVIVWT LVDINRRDPL VVAALHLCIA LGYANSSLNP VLYAFLDENF KRCFRQLCRT 361 409

RK IKMRMERQST NRVRNTVQDP ASMRDVGGMN KPV ........

GK IKMRMERQST SRVRNTVQDP AYMRNVDGVN KPV ........

GM TSSTIEQQNS TRVRQNTREH PSTANTVDR.

RM TSSTIEQQNS TRVRQNTREH PSTANTVDRT NHQLENLEAE TAPLP

Md PCGRQEPGSL RRPRQATTRE RVTACTPSDG PGGGRAA........

Legend: Rk: rat kappa opioid receptor (KOR), Gk: gP KÖ, Rm: rat mu opioid receptor (MOR), Gm: gp MOR, Md: mouse delta opioid receptor (DOR), Gd: gp DOR; bold letters AA differences: $\mathrm{Rk}$ vs. $\mathrm{Gk}, \mathrm{Rm}$ vs. $\mathrm{Gm}$, and Md vs. Gd; TM: transmembrane domains.

The cloned receptor fragments show that both the gp delta- and mu opioid receptor AAsequences are highly homologous to the rat and mouse opioid receptor sequences. Since we are particularly interested in the influence of opioids on the gastrointstinal motility, those clones may be used to investigate the distribution of opioid receptors across the gastrointestinal tract of guinea pig.

Thanks: This work was supported in part by the "Deutscher Akademischer Austauschdienst", Bonn, FRG.

References

1. Goldstein A, Naidu A (1989) Mol. Pharmacol. 36:265-272

2. Evans CJ, Keith DE, Morrison H, Magendzo K, Edwards RH (1992) Science 258:1952-1955

3. Kieffer BL, Befort K, Gaveriaux-Ruff C, Hirth CG (1992) Proc. Natl. Acad. Sci. USA 89: 12048-12052

4. Xie GX, Meng F, Mansour A, Thompson RC, Hoversten MT, Goldstein A, Watson SJ, Akil H (1994) Proc. Natl. Acad. Sci. USA 91: 3779-3783

5. Thompson RC, Mansour A, Akil H, Watson SJ (1993) Neuron 11:1-20 